\title{
Anomalías congénitas y comorbilidad en neonatos con Síndrome de Down
}

\section{Congenital anomalies and comorbidities in neonates with Down Syndrome}

\author{
Nicole Nakousi Capurro ${ }^{a}$, Carolina Cares Basualto ${ }^{b, c, d}$, Angélica Alegría Olivos ${ }^{e}$, \\ Marina Gaínza Lein ${ }^{\mathrm{f}, \mathrm{g}}$, Luis López Aristizabal ${ }^{\mathrm{h}}$, Alejandro Gayan Torrente $\mathrm{i}^{\mathrm{i}, \mathrm{j}}$, \\ Valentina Ojeda Contrerask, María José Irarrázaval Montero ${ }^{k}$
}

\author{
aBecaria, Programa de Especialización en Genética Clínica. Sección de Genética Clínica, Hospital Clínico Universidad de Chile, Santiago, Chile \\ bServicio de Genética Clínica Hospital Luis Calvo Mackenna, Santiago, Chile \\ 'Servicio de Genética Clínica Dávila, Santiago, Chile. Santiago, Chile \\ 'Laboratorio de Citogenética Clínica Dávila, Santiago, Chile \\ eServicio de Neonatología Clínica Dávila, Santiago, Chile \\ fInstituto de Pediatría, Facultad de Medicina, Universidad Austral de Chile. Valdivia, Chile \\ 9Servicio de Neuropsiquiatría Infantil, Hospital Clínico San Borja Arriarán, Universidad de Chile, Santiago, Chile \\ hBecario, Programa de Especialización en Pediatría, Universidad Diego Portales. Servicio de Pediatría, Clínica Dávila. Santiago, Chile \\ iServicio Cardiología Infantil Hospital Roberto del Rio. Santiago, Chile \\ Servicio Cardiología Infantil, Clínica Dávila. Santiago, Chile \\ kEstudiante de $7^{\circ}$ año de Medicina, Escuela de Medicina, Universidad de Los Andes. Santiago, Chile
}

Recibido: 22 de noviembre de 2019; Aceptado: 21 de julio de 2020

¿Qué se sabe del tema que trata este estudio?

El Síndrome de Down se asocia a múltiples anomalías congénitas y morbilidades. Los recién nacidos con Síndrome de Down suelen presentar menor peso y semanas de edad gestacional al nacimiento, aumentando su riesgo de comorbilidades neonatales.
¿Qué aporta este estudio a lo ya conocido?

Se presenta el primer reporte de una serie neonatal chilena con Síndrome de Down desde el año 2009. La información de este trabajo demuestra la necesidad de una atención multidisciplinaria guiada por protocolos de manejo comprensivos y estandarizados.

\section{Resumen}

El Síndrome de Down se presenta en 2,5 de 1.000 recién nacidos vivos chilenos. Presentan más anomalías congénitas y comorbilidades que la población general, aumentando su tasa de hospitalización. Objetivo: Describir las anomalías congénitas y comorbilidades de neonatos con Síndrome de Down nacidos y/u hospitalizados en la década 2008-2018. Pacientes y Método: Retrospectivamente se revisaron registros de los pacientes nacidos y/u hospitalizados dentro de sus 28 días de vida entre el 1 de enero de 2008 y el 31 de diciembre de 2018. Para cada paciente se consignó: edad
Palabras clave:

Síndrome de Down;

Trisomía 21;

Morbilidad Neonatal;

Cuidado Intensivo

Neonatal;

Anomalías Congénitas

Correspondencia:

Nicole Nakousi Capurro

sandra.nakousi@ug.uchile.cl 
materna, antecedentes familiares de Síndrome de Down, antecedentes pre y perinatales y resultado de estudio genético. Se consignó la edad al ingreso, el motivo principal de ingreso, comorbilidades, días de hospitalización y fallecimiento. Se excluyeron dos pacientes con más del $50 \%$ de ficha incompleta. Se exploraron asociaciones entre morbilidades, anomalías y fallecimiento. Resultados: 140 de $79.506(0,2 \%)$ recién nacidos vivos fueron diagnosticados con Síndrome de Down en el período neonatal. $24,7 \%$ fueron prematuros y $26,4 \%$ tuvieron bajo peso para su edad gestacional. Los porcentajes de morbilidad y hospitalización fueron $83,6 \%$ y $90 \%$. La principal causa de ingreso fue la poliglobulia, y la más frecuente hiperbilirrubinemia. Fallecieron 4 pacientes $(2,9 \%)$ y $70,7 \%$ presentó alguna una anomalía congénita, principalmente cardíaca. La mediana de edad materna fue de 36 años y 57,1\% tenía 35 años o más. Conclusiones: Esta investigacion aporta información relevante para optimizar el manejo perinatal y el seguimiento de los pacientes con Síndrome de Down.

\begin{abstract}
In Chile, Down syndrome has a prevalence of 2.5 in 1,000 live births. These patients present more congenital anomalies and comorbidities than the general population, increasing their hospitalization rate. Objective: To describe congenital anomalies and comorbidities of neonates with Down syndrome born and/or hospitalized between 2008 and 2018. Patients and Method: We conducted a retrospective review of patient's medical records born and/or hospitalized during their first 28 days of life between January $1^{\text {st }}, 2008$, and December $31^{\text {st }}, 2018$. For each patient, we recorded maternal age, familiar cases of Down Syndrome, pre and perinatal history, genetic study result, as well as age at admission, reason for hospitalization, comorbidities, length of stay, and death. Two patients that had more than $50 \%$ of incomplete medical records were excluded. We studied the associations between comorbidities, congenital anomalies, and death. Results: 140 in 79,506 newborns $(0.2 \%)$ were diagnosed at our center with Down Syndrome in their neonatal period. $24.7 \%$ were born preterm and $26.4 \%$ had low birth weight for gestational age. Morbidities and hospitalizations were present in $83.6 \%$ and $90 \%$, of the study population, respectively. The main reason for hospitalization was polycythemia and the most frequent was hyperbilirubinemia. Four patients died $(2.9 \%)$ and $70.7 \%$ presented at least one congenital anomaly, mainly heart disease. Median maternal age was 36 years and $57.1 \%$ of mothers were aged 35 or older. Conclusions: This cohort of patients with Down Syndrome provides important information for the optimization of their perinatal management and follow-up.
\end{abstract}

Keywords:

Down syndrome;

Trisomy 21;

Neonatal Morbidity;

Neonatal Intensive

Care;

Congenital Anomalies

\section{Introducción}

El Síndrome de Down (SD) es una cromosomopatía frecuente, encontrándose en 1 de 1.000 recién nacidos vivos $(\mathrm{RNV})^{1}$. De acuerdo al Estudio Colaborativo Latinoamericano de Malformaciones Congénitas (ECLAMC) del período 2001-2010, en Chile se estima una tasa de 2,5 por $1.000 \mathrm{RNV}^{2}$. Considerando la natalidad nacional nacerían aproximadamente 600 pacientes con SD al año en Chile ${ }^{3}$. Su frecuencia ha ido en aumento en nuestro país, lo que no se explicaría únicamente por el aumento de la edad materna, pero hasta ahora no se han comprobado otras causas ${ }^{4}$.

Los pacientes con SD presentan mayores índices de anomalías congénitas (AC) que la población general, particularmente cardíacas (54-66\%) y digestivas (3-13\%). Al nacimiento pueden pesar menos que RN sanos y presentar hiperbilirrubinemia, hipoglicemia y distrés respiratorio, lo que junto a lo anterior eleva las tasas de hospitalización respecto a la población general 2 a 5 veces, particularmente en el primer año y mes de vida $^{5-11}$. La detección precoz de AC (particularmente cardiopatías) mejora el pronóstico de estos pacientes significativamente ${ }^{9,12}$.

En el período 2008-2018, la Clínica Dávila de Santiago atendió un promedio de 7.200 partos anuales, correspondiente al 3\% de los partos nacionales ${ }^{3}$. La población consultante incluye población chilena e inmigrante, del sector público y privado de salud.

Existen pocos estudios que describan neonatos chilenos con SD, y no hay reportes desde hace más de 10 años $^{2,4,6,13,14}$. Al ser una cantidad considerable los pacientes atendidos en nuestro centro, esta cohorte representa una oportunidad para describir las manifestaciones clínicas de este síndrome y ofrecerles una atención adecuada y oportuna.

El objetivo de este estudio es describir la morbilidad y AC en neonatos con SD nacidos y/u hospitalizados en nuestro centro entre 2008-2018. 


\section{Pacientes y Método}

\section{Tipo y población de estudio}

Se revisaron retrospectivamente los registros clínicos electrónicos y físicos de pacientes con diagnóstico de SD en su egreso de puerperio o Neonatología, nacidos en la clínica y/u hospitalizados en el Servicio de Neonatología, Clínica Dávila de Santiago de Chile, entre 1 de enero de 2008 y el 31 de diciembre de 2018. Se consignó información de los primeros 28 días de vida en un registro por paciente, considerando reingresos en ese período. Se excluyeron dos casos con más del $50 \%$ de su ficha incompleta. No se realizó seguimiento a los pacientes luego del alta.

Para el cálculo de tasa de nacimiento de SD se consideraron todos los recién nacidos vivos (RNV) en nuestro centro, incluyendo a aquellos que fueron trasladados posteriormente y se excluyeron a los que llegaron derivados para hospitalización desde otras instituciones. Para el análisis de AC y morbilidad se consideraron todos los casos (nacidos con o sin derivación posterior, y aquellos que ingresaron desde otros centros).

\section{Variables}

Epidemiológicas. Se incluyeron antecedentes familiares con $\mathrm{SD}$, sospecha ecográfica prenatal, diagnóstico citológico prenatal, edad gestacional (EG) y adecuación de peso según curvas de Alarcón-Pittaluga ${ }^{15}$. Se consignó el resultado del estudio genético solicitado e información relacionada a hospitalización, incluido fallecimiento intrahospitalario y su causa.

Morbilidad. El diagnóstico de poliglobulia se realizó con hematocrito venoso central mayor o igual a $65 \%{ }^{16}$. La hiperbilirrubinemia se diagnosticó con una bilirrubinemia capilar sobre la curva de riesgo para fototerapia de la Academia Americana de Pediatría para RN mayores de 35 semanas y para menores de 35 semanas se usaron las curvas de las guías británicas $\mathrm{NICE}^{17,18}$. Se constató hipoglicemia con glicemias bajo $47 \mathrm{mg} / \mathrm{dL}(2,6 \mathrm{mmol} / \mathrm{L})$ evaluados por cinta reactiva en hemoglucotest ${ }^{19,20}$. El hipotiroidismo congénito se diagnosticó con dos mediciones seguidas de TSH plasmática mayor o igual a $10 \mathrm{mU} / \mathrm{L}$ y con un valor de T4 libre bajo ${ }^{21}$. Se diagnosticó Síndrome de Distrés Respiratorio por clínica compatible y requerimientos de oxígeno; hipertensión pulmonar según definición clínica y ecocardiografía ${ }^{22}$; trombocitopenia se consideró con recuento de plaquetas menor a 150 por $10^{923}$.

Anomalías congénitas. Se consignaron en todos los sistemas. En el cardíaco se consideraron todos los hallazgos ecocardiográficos, que incluían anomalías estructurales. Se incluyó el Ductus Arterioso persis- tente (DAP) porque el tiempo de seguimiento no permitió conocer su desenlace. No se incluyeron: a) DAP en $\mathrm{RN}$ menores de 32 semanas o en vías de cierre, $\mathrm{b}$ ) comunicación interauricular (CIA) o interventricular (CIV) mínima o en vías de cierre, c) hallazgos aislados inespecíficos. Se agruparon como "defectos atrioventriculares" (DAV) a los canales atrioventriculares y a pacientes con coexistencia de CIV y CIA, por considerarse estados tardíos de cierre de un canal auriculoventricular. Se clasificó "Tetralogía de Fallot" cuando se objetivaron los cuatro hallazgos ecocardiográficos en conjunto, cuantificándose como un solo defecto cardíaco, de lo contrario se registraron los defectos ais$\operatorname{lados}^{24}$.

Los datos fueron revisados por una Neonatóloga, Cardiólogo Pediatra, Residente de Pediatría y Genetista Clínico.

\section{Análisis estadístico}

Las variables categóricas se expresaron como número total y porcentajes. Las variables continuas como media, mediana, p25-p75 y rango.

La asociación entre total de morbilidades/AC y fallecimiento se calculó a través de modelos de regresión logística, así como también la asociación entre la presencia de AC y fallecimiento. La asociación entre sistema afectados por AC se analizó con prueba exacta de Fisher. Se consideró significativo un valor alfa de 0,05. Todos los análisis se realizaron con Stata, versión 14.2 (StataCorp LLC).

\section{Consideraciones éticas}

Este trabajo contó con la aprobación del Comité de Ética de la Clínica Dávila de Santiago para la revisión de fichas clínicas y registros electrónicos.

\section{Resultados}

Durante el período 2008-2018 nacieron 79.506 RNV en nuestro centro, de los cuales 140 fueron diagnosticados con SD durante periodo neonatal. Las tasas anuales por cada 1.000 RNV oscilaron entre 1,4 y 2,4 (en los años 2009 y 2018, respectivamente). No se observó una tendencia al aumento o disminución de esta durante el período estudiado; las tasas más elevadas se encontraron en los años 2008 y 2018 (2,2 y 2,4 respectivamente). La tasa para todo el período fue de 1,8 cada $1.000 \mathrm{RNV}$.

Dos pacientes (uno de cada sexo) nacidos en nuestro centro fueron excluidos de la muestra por registros clínicos incompletos. Dos pacientes fueron trasladados al Servicio de Neonatología (a los 7 y 10 días de vida) y se consideraron para el análisis de $\mathrm{AC}$ y morbilidades.

La descripción general de la población se encuentra 
en la tabla 1 . Destaca un $24,7 \%$ de prematurez y un $26,4 \%$ de bajo peso para la EG. Dieciocho pacientes (12,9\%) cumplieron ambas condiciones. Casi la totalidad de los casos exhibió un puntaje de Apgar normal a los 5 minutos. La mediana de morbilidad en RN de término fue de 1 , y en prematuros fue de 2. La mediana de AC en ambos grupos fue 1.

La mediana (p25-75) de edad materna fue de 36 años (31-39) con un porcentaje de edad mayor o igual a 35 años de $57,1 \%$. El porcentaje de madres con 40 años o más fue de $24,3 \%$.

La tasa de hospitalización fue de $90 \%$, con una mediana de edad al ingreso de 1 día de vida y 6 días de estancia. Los principales motivos de ingreso se encuentran en la tabla 2.

Un $83,6 \%$ de pacientes (117/140) presentó morbilidad y fuera de un caso, todos fueron hospitalizados. El principal motivo de ingreso fue la poliglobulia (26/126 hospitalizados) (tabla 2), pero la morbilidad más prevalente durante el primer mes fue la hiperbilirrubinemia (tabla 3 ).

Fallecieron cuatro pacientes antes del mes de vida (2,9\%)

Tabla 1. Descripción general de la población muestral

\begin{tabular}{ll}
\hline Total & 140 \\
\hline Género & \\
Masculino N (\%) & $83(59,3)$ \\
Femenino N (\%) & $1(0,7)$ \\
Indefinido N (\%) & \\
Semanas de edad gestacional & $38(37-39)$ \\
Mediana (p25-75) & $35(24,7)$ \\
Edad gestacional < 37 semanas N (\%) & \\
Peso de nacimiento & \\
Adecuación según edad gestacional N $(\%)$ \\
AEG & $96(68,6)$ \\
PEG & $37(26,4)$ \\
GEG & $7(5)$ \\
Peso en gramos: mediana (p25-75) & $3.015(2.510-3.360)$ \\
Puntaje de Apgar & \\
1 minuto $\leq 6:$ N (\%) & $12(8,6)$ \\
5 minutos $\leq 6:$ N (\%) & $2(1,4)$ \\
Sospecha ecográfica prenatal &
\end{tabular}

AEG: adecuado para edad gestacional. PEG: pequeño para edad gestacional. GEG: grande para edad gestacional. durante la hospitalización neonatal en nuestro centro. Un paciente de 36 semanas de EG por DAV severo y sepsis, otra de 32 semanas por sepsis, otro de 34 semanas por AC cardíacas múltiples, laringotraqueomalacia e infección respiratoria y otro por prematurez extrema (24 semanas), malformación cardíaca y complicaciones hematológicas. Dos de ellos fueron pequeños para edad gestacional (PEG).

A 70,7\% de los RN con SD se les detectó alguna AC. Se observó compromiso cardíaco en $64,3 \%$ de la población y el hallazgo ecocardiográfico acompañante más frecuente fue el foramen oval permeable, en un 18,6\%. Se observó una AC en los sistemas gastrointestinal, genitourinario y de pared abdominal en el 9,3\%, 6\% y 3,3\% de la muestra, respectivamente (tabla 4). La mediana (p25-75) de AC por paciente fue de $1(0-2)$. Nuestra población presentó ninguna $(29,3 \%)$, dos $(40,7 \%)$ y tres $(22,1 \%)$ o más $(7,9 \%)$ AC.

Veintiocho $(19,9 \%)$ pacientes tuvieron sospecha prenatal ecográfica de SD, de los cuales dos $(7,1 \%)$ se confirmaron con cariograma prenatal.

Se realizó estudio genético en 127 pacientes (90\% de la muestra). Un estudio correspondió a una inmunofluorescencia in situ (FISH) con sondas para cromosoma 21 en sangre y sin confirmación posterior con cariograma, por lo que no se incluyó en la caracterización citogenética de la tabla 5.

Tabla 2. Datos de pacientes con Síndrome de Down hospi-
talizados en Neonatología, principal motivo de ingreso y tasa de fallecimiento

\begin{tabular}{ll}
\hline Hospitalizados & $N=126$ \\
Edad de hospitalización, en días & $1(1-2)$ \\
Mediana (p25-75) & $0-27$ \\
Rango & $6(4-11)$ \\
Días hospitalizados: mediana (p25-75) & $N(\%)$ \\
Principal motivo de hospitalización & $26(18,6)$ \\
Poliglobulia & $22(15,7)$ \\
Distrés respiratorio & $17(12,1)$ \\
Hiperbilirrubinemia & $14(10)$ \\
Hipoglicemia & $13(9,3)$ \\
Cardiopatía congénita & $11(7,9)$ \\
Malformación tracto digestivo & $7(5)$ \\
Bajo peso de nacimiento & $6(4,3)$ \\
Prematurez & $4(2,9)$ \\
Hipertensión pulmonar & $6(4,3)$ \\
Otros: & $1(0,7)$ \\
Cianosis & $1(0,7)$ \\
Onfalitis & $1(0,7)$ \\
Dificultad para alimentarse & $1(0,7)$ \\
Estudio hidrops fetal & $1(0,7)$ \\
Hipotermia obs infección connatal & $1(0,7)$ \\
No especificada & $4(2,9)$ \\
Fallecen en hospitalización N (\%) & $14 / 140(10)$ \\
No hospitalizados N (\%) &
\end{tabular}




\begin{tabular}{|c|c|}
\hline Morbilidad & N (\%) \\
\hline Hiperbilirrubinemia & $68(48,6)$ \\
\hline Por incompatibilidad de grupo sanguíneo & $9(6,4)$ \\
\hline Poligobulia & $43(30,7)$ \\
\hline Distrés respiratorio & $35(25)$ \\
\hline Taquipnea transitoria & $15(10,7)$ \\
\hline Hipertensión pulmonar persistente & $10(7,1)$ \\
\hline Enfermedad membrana hialina & $4(2,9)$ \\
\hline Cardiopatía & $3(2,1)$ \\
\hline Poliglobulia & $1(0,7)$ \\
\hline Quilotórax congénito bilateral & $1(0,7)$ \\
\hline No especificado & $1(0,7)$ \\
\hline Hipoglicemia & $27(19,3)$ \\
\hline Trombocitopenia & $18(12,9)$ \\
\hline Anemia & 7 (5) \\
\hline Hipotiroidismo & $5(3,6)$ \\
\hline Infección respiratoria & $5(3,6)$ \\
\hline Infección oftalmológica & $5(3,6)$ \\
\hline Hipocalcemia & $4(2,9)$ \\
\hline Shock séptico & $4(2,9)$ \\
\hline Infección tracto urinario & $2(1,4)$ \\
\hline Otros & 6 \\
\hline Trastorno mieloproliferativo transitorio & $1(0,7)$ \\
\hline Onfalitis & $1(0,7)$ \\
\hline Rectorragia & $1(0,7)$ \\
\hline Enfermedad hemolítica & $1(0,7)$ \\
\hline Sífilis congénita & $1(0,7)$ \\
\hline Hipocalemia & $1(0,7)$ \\
\hline Morbilidades por paciente: mediana (p25-75) & $2(1-2)$ \\
\hline Total sin morbilidad N (\%) & $23(16,4)$ \\
\hline
\end{tabular}

\section{Análisis de asociaciones}

No hubo asociación significativa entre mortalidad neonatal y número de morbilidades (OR 1,8; $\mathrm{p}=0,1$; CI 95\% 1-3,5) ni número de AC (OR 1,2; $\mathrm{p}=0,7$; CI 95\% 0,4-3,5).

Los RN prematuros presentaron una mediana de 2 morbilidades y los de término 1 , sin diferencia: OR 1,4 ( $\mathrm{p}=0,1$; $95 \%$ CI 1-1,9). No se demostró diferencia en número de AC entre ambos grupos.

No fue posible analizar asociaciones entre los dos casos con diagnóstico de Trisomía 21 en mosaico y otras variables por encontrarse sólo 2 casos que difirieron ampliamente entre sí.

De los pacientes con compromiso cardíaco, 8/90 (8,9\%) presentaron también afectación del sistema gastrointestinal. Del total de pacientes con anomalías gastrointestinales, 8/13 $(61,5 \%)$ demostraron compromiso cardíaco concomitante.
Tabla 4. Anomalías congénitas detectadas en período neonatal según sistema

\begin{tabular}{|c|c|}
\hline$N=140$ & N (\%) \\
\hline $\begin{array}{l}\text { Sistema Cardíaco } \\
\text { Total de pacientes afectados } \\
\text { Defectos aurículo-ventriculares } \\
\text { CIA } \\
\text { CIV } \\
\text { Tetralogía de Fallot } \\
\text { Displasia valvular } \\
\text { Cabalgamiento de Aorta } \\
\text { Insuficiencia tricuspídea y estenosis pulmonar } \\
\text { DAP }\end{array}$ & $\begin{array}{cc}90 & (64,3) \\
28 & (20) \\
22 & (15,3) \\
17 & (11,3) \\
3 & (2) \\
2 & (1,3) \\
1 & (0,7) \\
1 & (0,7) \\
47 & (31,3)\end{array}$ \\
\hline $\begin{array}{l}\text { Sistema Gastrointestinal } \\
\text { Total } \\
\text { Ano imperforado } \\
\text { Atresia esofágica } \\
\text { Atresia duodenal } \\
\text { Enfermedad de Hirschprung } \\
\text { Estenosis duodenal }\end{array}$ & $\begin{aligned} 13 & (9,3) \\
4 & (2,7) \\
3 & (2) \\
3 & (2) \\
2 & (1,3) \\
1 & (0,7)\end{aligned}$ \\
\hline $\begin{array}{l}\text { Sistema Genitourinario } \\
\text { Total } \\
\text { Criptorquidia unilateral } \\
\text { Criptorquidia bilateral } \\
\text { Displasia renal }\end{array}$ & $\begin{array}{ll}9 & (6) \\
5 & (3,3) \\
3 & (2) \\
1 & (0,7)\end{array}$ \\
\hline $\begin{array}{l}\text { Sistema Pared abdominal } \\
\text { Total } \\
\text { Hernia umbilical } \\
\text { Hernia inguinal }\end{array}$ & $\begin{array}{ll}5 & (3,3) \\
3 & (2) \\
2 & (1,3)\end{array}$ \\
\hline $\begin{array}{l}\text { Sistema Respiratorio } \\
\text { Total } \\
\text { Laringotraqueomalacia }\end{array}$ & $\begin{array}{ll}1 & (0,7) \\
1 & (0,7)\end{array}$ \\
\hline
\end{tabular}

CIA: comunicación interauricular. CIV: comunicación interventricular. DAP: ducto arterioso persistente.

Tabla 5. Descripción citogenética de pacientes nacidos en nuestro centro con Sindrome de Down

\begin{tabular}{lrc}
\hline Total $=140$ & $N \quad(\%)$ \\
\hline Antecedentes familiares consignados & & \\
Casos familiares previos de SD & $(2,9)$ \\
Sin casos familiares previos de SD & $60(42,9)$ \\
Sin antecedentes & $76(54,3)$ \\
Cariograma & \\
En linfocitos (post natal) & $124 / 140(88,6)$ \\
En amniocitos (pre natal) & $1 / 140 \quad(0,7)$ \\
En vellosidad corial (pre natal) & $1 / 140 \quad(0,7)$ \\
Resultados disponibles: & $125 / 126$ \\
Trisomía 21 libre & $119 / 125(95,2)$ \\
Trisomía 21 por traslocación & $4 / 125 \quad(3,2)$ \\
Trisomía 21 en mosaico & $2 / 125 \quad(1,6)$ \\
Otros estudios genéticos & & \\
FISH cr 21 en linfocitos & $1 / 140 \quad(0,7)$ \\
Sin antecedentes & $12 / 140 \quad(8,6)$ \\
\hline
\end{tabular}

FISH: Hibridización fluorescente in-situ, con sondas para cromosoma 21. 
No se encontró una asociación significativa entre la presencia de AC cardíacas y gastrointestinales (OR 1,2; $\mathrm{p}=0,7 ; 95 \%$ CI 0,4-3,5).

\section{Discusión}

Describimos a una población de pacientes con SD nacidos u hospitalizados durante su primer mes de vida en una clínica entre los años 2008-2018, en donde se observó una tasa de nacimiento de 1,8 pacientes con SD cada 1.000 RNV, con una tendencia estable en ese período. Esta tasa fue inferior a lo reportado en series chilenas en años anteriores y es levemente mayor que las últimas tasas de Latinoamérica ${ }^{2,4,25}$.

La mayoría de pacientes fueron de sexo masculino, con un peso apropiado a su EG y un adecuado puntaje de Apgar a los 5 minutos. La mayoría de los pacientes nació de término y un $24,7 \%$ fue prematuro, superando por mucho al $8 \%$ reportado en RN chilenos ${ }^{26}$. Esta tendencia replica la de otra serie nacional, donde el 21,2\% de 33 pacientes fue prematuro y sería concordante con el 9-30\% de prematurez en series internacionales ${ }^{5,727-30}$. No se sabe claramente por qué se observa mayor tasa de prematurez en RN con SD. Se ha descrito una asociación entre defectos congénitos y embarazos más cortos en población general, la que podría explicar un porcentaje de nuestros $\operatorname{casos}^{31,32}$. Observamos una prevalencia de $\mathrm{AC}$ en nacimientos prematuros de $74,3 \%$ y en los de término: $67,6 \%$, diferencia no significativa. Especulamos que también podrían existir elementos en la población con SD que lleven a una inducción más prematura del parto como la detección de AC, ruptura precoz de membranas o retraso del crecimiento intrauterino, lo que sería interesante explorar a futuro ${ }^{33,34}$.

El RN con SD también se asocia a un peso de nacimiento inferior al de la población general ${ }^{28,30,35}$. Nuestro 26,4\% fue inferior al 30\% descrito en otra cohorte chilena y superior a otra con $12 \%$ de pacientes con peso menor a 2.000 g y EG promedio de 37,3 semanas $^{6,13}$. Esto se explicaría por la constitución genética del individuo, las AC y factores placentarios y uterinos $^{31-34}$

El principal factor de riesgo para tener SD es una mayor edad materna, y en nuestra población encontramos una edad materna con una mediana de 36 años. Al nacer, más de la mitad de los pacientes con SD tuvieron madres con edades iguales o superiores a 35 años y un grupo importante tenía 40 años o más (23,9\%). Sólo otro estudio chileno reporta un $51,5 \%$ de casos con SD y madres de 35 años o más, difiriendo de otros en donde el porcentaje de madres sobre los 35 años es significativamente inferior ${ }^{4,6,36}$. El porcentaje de niños con SD con madres mayores a 35 años ha aumentado, lo que podría deberse al aplazamiento de la materni- dad en Chile o un eventual aumento de edad de las pacientes atendidas en nuestro centro ${ }^{37,38}$. El segundo factor de riesgo para SD es la presencia de una traslocación cromosómica balanceada en un progenitor que segregaría en la formación de gametos causando una trisomía 21 por traslocación, antecedentes familiares de SD o pérdidas reproductivas. Este tipo de trisomía se observó en cuatro pacientes, sin antecedentes familiares.

A nivel internacional la opción de interrupción del embarazo influye en la tasa de nacimiento de $\mathrm{RN}$ con $\mathrm{SD}$. Esto no afecta nuestras cifras por no existir en Chile la interrupción del embarazo por presentar $\mathrm{SD}^{36,37}$.

Las hospitalizaciones neonatales difirieron de series nacionales e internacionales en su tasa y motivos de ingreso. El 90\% de la muestra ingresó al Servicio de Neonatología, mayoritariamente en su primer día de vida. Esta sería similar al 84,8\% de otra serie chilena con 33 $\operatorname{casos}^{6}$. Nuestra tasa de hospitalización se asemejaría a las tasas más altas reportadas en países europeos ${ }^{27}$. La mediana de hospitalización fue de 6 días. El principal motivo de ingreso fue la poliglobulia $(18,6 \%)$, lo que contrasta con otras series donde la principal causa de ingreso es el distrés respiratorio. Este fue nuestro segundo motivo de ingreso $(15,7 \%)$ y en tres casos fue secundaria a cardiopatía congénita. En la serie chilena de Retamales et al, la dificultad respiratoria se registró en el 7\% como el principal motivo de admisión, separado de las cardiopatías congénitas, y en series internacionales se reporta en un $32-72 \%$ de casos. Este porcentaje probablemente varía según el diseño de cada estudio. La hiperbilirrubinemia suele ser la segunda causa de ingreso en estos pacientes luego de las AC y su prevalencia varía entre el $23-14 \%$, similar a lo observado en nuestra serie $\mathrm{e}^{6,28}$.

El 83,6\% de nuestra población presentó al menos una morbilidad neonatal y la mayoría correspondió a patología frecuente del RN sin SD. Explicaciones para esta cifra incluyen: a) el mayor porcentaje de estos pacientes estuvo hospitalizado $(82,9 \%)$ por lo que podría corresponder a una sobreestimación de la morbilidad, y b) una alta prevalencia de pacientes PEG y prematuros, lo que aumenta el riesgo de patologías concomitantes.

Una importante causa de hospitalización neonatal en pacientes con SD son las AC y en nuestra serie conforman el segundo motivo de ingreso más frecuente en conjunto $(17,1 \%)^{10}$. Durante su estancia hospitalaria, a un 70,7\% de pacientes se le detectó al menos una AC, y $64,3 \%$ presentó al menos una anomalía cardía$\mathrm{ca}$, en congruencia con el 54-66\% de prevalencia en $\mathrm{RN}$ con $\mathrm{SD}^{8}$. Se incluyeron aquellos DAP significativos en recién nacidos mayores de 32 semanas porque se asocian a $\mathrm{SD}^{36-38}$. Aunque esto podría sobreestimar las cardiopatías, sólo seis pacientes presentaron DAP 
como única anomalía cardíaca, de los cuales tres la presentaron como AC única, por lo que creemos que no altera significativamente las tasas. Los DAV son la AC cardíaca más frecuente en SD (prevalencia aproximada: $42 \%)^{8,27,41,42}$. Al igual que otra serie chilena, observamos una frecuencia de DAV menor a la internacional $\left(25 \%\right.$ y $20 \%$, respectivamente) ${ }^{6}$. Sin embargo, y a diferencia de reportes nacionales e internacionales, observamos más CIA que $\mathrm{CIV}^{6,8}$. Los tipos de anomalías observadas en tubo digestivo y su tasa de presentación $(7,9 \%)$ estuvieron acorde a lo descrito en otras series ${ }^{6,28,43}$.

Existen dificultades para comparar nuestros tipos y frecuencias de AC con otros estudios. En primer lugar, existen pocas series nacionales que reporten los mismos datos neonatales en una población de $\mathrm{RN}$ con $\mathrm{SD}$, a excepción de Retamales et $\mathrm{al}^{6}$. En segundo lugar, existen grandes diferencias metodológicas entre series en el método de comprobación de AC, tiempo de seguimiento, criterios de inclusión/exclusión, y número de $\operatorname{casos}^{43}$. Finalmente, a nivel internacional existen variados criterios de interrupción del embarazo. Esto puede llevar a todo tipo de divergencias en resultados, especialmente en AC. Por ejemplo, la incidencia y tipo de cardiopatías -especialmente las complejas- son más frecuentes en países sin detección prenatal o sin acceso a terminación legal del embarazo, llegando hasta $60 \%{ }^{8,27,41}$. Además, en países con screening prenatal y terminación del embarazo la prevalencia de CIV y canal auriculoventricular comienzan a parecerse entre sí $(30 \% \text { a } 31 \% \text {, respectivamente })^{41}$.

No se demostró significancia estadística en la asociación entre sistemas con AC. Eso se ha reportado para sistemas cardíaco y gastrointestinal en población sin cromosomopatías, pero aún no significativamente en $\mathrm{SD}^{41}$. De todas formas se pesquisó una AC cardíaca en el $63,6 \%$ de pacientes con alguna anomalía gastrointestinal.

Nuestra tasa de mortalidad neonatal fue de 2,6\%, levemente menor al 3,7-6\% descrito ${ }^{27,28}$. Durante este período fallecieron cuatro pacientes; dos tuvieron bajo peso de nacimiento, tres presentaron una cardiopatía congénita y todos fueron prematuros, en concordancia con los principales factores de riesgo de mortalidad neonatal en SD. Otros factores incluyen etnia, aspectos socioeconómicos y educación materna ${ }^{7,10,12}$. Datos chilenos muestran que la mayor tasa de mortalidad de pacientes con SD es en el período post neonatal ${ }^{6,44}$.

El SD puede sospecharse prenatalmente por marcadores ecográficos de aneuoploidías con o sin tests citológicos, AC y/o screening de ADN fetal libre en plasma materno $\mathrm{o}^{45}$. La confirmación diagnóstica se realiza a través de cariograma en tejido fetal. Del total de fichas con información prenatal disponible $(\mathrm{N}=82)$, la mayoría no tenía sospecha ecográfica de SD y sólo dos fueron confirmados por cariograma prenatal. Cabe destacar que dentro de ese $41 \%$ sin antecedentes podrían encontrarse casos con y sin sospecha. Desconocemos dónde los pacientes realizaron su seguimiento prenatal y qué factores influyeron en la ausencia de ese dato en las fichas de Neonatología/Puerperio. A pesar de no ser uno de nuestros objetivos, creemos que sería interesante completar esta información y analizar estos y otros aspectos de la sospecha prenatal en otro trabajo.

A pesar de contar con sólo 125 resultados de cariograma, se mantuvo la distribución esperada de tipos de anomalías citogenéticas en SD: 95\% trisomía libre (95,2\%), 3\% por traslocación Robertsoniana u otro rearreglo cromosómico (3,2\%), y $2 \%$ mosaico $(1,6 \%)$ (46). El cariograma en linfocitos ayuda a establecer el diagnóstico y riesgo de recurrencia de SD. En una trisomía libre el riesgo de recurrencia depende del número previo de embarazos con SD de la pareja y edad materna. Este riesgo basalmente se considera bajo el $1 \%$, pero va aumentando con la edad. En presencia de una trisomía 21 por traslocación, el riesgo de recurrencia puede variar del 1 al 100\%, dependiendo del tipo de traslocación y el progenitor que la porte, por lo que en estos casos siempre deben estudiarse los padres ${ }^{46}$. El estudio FISH no ayuda a distinguir entre una trisomía libre o por traslocación, y a pesar ser un examen más rápido no permite hacer una consejería adecuada si no se acompaña de un cariograma.

El tamaño de la muestra no otorgó un adecuado poder estadístico para explorar otras asociaciones de mortalidad neonatal, morbilidad y AC con otras variables. La falta de información del control ecográfico prenatal no permitió establecer asociaciones significativas con variables prenatales. Otras debilidades del trabajo son su carácter retrospectivo y sesgo en la selección y categorización de diagnósticos de ingreso, morbilidades y AC por nuestro equipo. Además, el tiempo de seguimiento impide estimar adecuadamente las cardiopatías congénitas al no filtrar a aquellos DAP no fisiológicos ni reportar adecuadamente otras AC y morbilidades detectables más adelante.

Entre las fortalezas encontramos un seguimiento de 10 años, en un centro con numerosos partos anuales y poca pérdida de casos.

Dada la alta incidencia pacientes con SD en nuestro país, en centros con gran cantidad de partos sería eventualmente beneficioso conformar un protocolo multidisciplinario que acompañe a la mujer embarazada y luego al binomio madre e hijo durante la sospecha, diagnóstico y seguimiento del síndrome. Este grupo debería estar integrado por especialistas en Obstetricia, Matronería, Neonatología, Pediatría, Genética, Kinesiología y otros según las necesidades de cada paciente. 


\section{Conclusiones}

Esta serie describe las principales características de pacientes chilenos con SD en el periodo neonatal desde el año 2009 al 2018. La tasa de nacimiento fue de 1,8 cada $10.000 \mathrm{RNV}$, inferior a la de otras series chilenas. Los pacientes presentaron una mayor tasa de prematurez y bajo peso, comorbilidad y tipos de AC. Se observó una elevada tasa de morbilidad y hospitalización neonatal, debida en su mayoría a patología frecuente del RN sin SD. La segunda causa de hospitalización fueron las AC, principalmente las cardíacas, seguidas por las gastrointestinales. La información de este estudio es relevante para una mejor organización del manejo perinatal de estos pacientes y sus múltiples complicaciones.

\section{Agradecimientos}

Los autores desean agradecer el apoyo de los tecnólogos médicos Pamela Arroyo y Dominique Chrzanowsky. Agradecemos también al Dr. Rodrigo Bancalari por su orientación endocrinológica. Por su asesoría agradecemos a los Ginecobstetras Especialistas en Medicina Materno Fetal: Dra. Paula Vergara y Dr. Nicolás Riquelme.

\section{Conflicto de intereses}

Los autores declaran no tener conflicto de intereses.

\section{Referencias}

1. WHO $\mid$ Genes and human disease [Internet]. WHO. [citado 3 de abril de 2016]. Disponible en: http://www.who. int/genomics/public/geneticdiseases/en/ indexl.html

2. Nazer J, Cifuentes L. Prevalencia al nacimiento de malformaciones congénitas en las maternidades chilenas participantes en el ECLAMC en el período 2001-2010. Rev Med Chile. 2014;142:1150-6.

3. Comité Nacional de Estadísticas Vitales. Serie histórica: Nacidos vivos observados, según región y provincia de residencia habitual de la madre, período 2005-2017. Instituto Nacional de Estadística (INE), Ministerio de Salud de Chile (MINSAL); 2018.

4. Nazer J, Cifuentes L. Estudio epidemiológico global del síndrome de Down. Rev Chil Pediatr. 2011;82:105-12.

5. Dawson A, Cassell C, Oster M, et al. Hospitalizations and associated costs in a population-based study of children with Down Syndrome born in Florida: Hospital Costs of Children with Down Syndrome. Birt Defects Res A Clin Mol Teratol. 2014;100:826-36.

6. Retamales N, Moreno R, González A, Cerda J, Lizama M. Morbilidad y Mortalidad durante el Primer Año de Vida en Pacientes con Síndrome de Down. Rev Chil Pediatr. 2009;80:323-31.

7. Glasson EJ, Jacques A, Wong K, Bourke J, Leonard H. Improved Survival in Down Syndrome over the Last 60 Years and the Impact of Perinatal Factors in Recent Decades. J Pediatr. 2016;169:214-220.e1.

8. Lagan N, Huggard D, Mc Grane F, et al. Multiorgan involvement and management in children with Down syndrome. Acta Paediatr [E-Pub, Ahead of Print, Internet]. 2020 [citado 11 de abril de 2020]; Disponible en: https:// onlinelibrary.wiley.com/doi/abs/10.1111/ apa. 15153

9. Zhu JL, Hasle H, Correa A, et al. Hospitalizations among people with Down syndrome: A nationwide population-based study in Denmark. Am J Med Genet A. 2013;161(4):650-7.

10. Fitzgerald P, Leonard H, Pikora TJ, Bourke J, Hammond G. Hospital Admissions in Children with Down Syndrome: Experience of a PopulationBased Cohort Followed from Birth. PLoS ONE. 2013;8:e70401.

11. Nazer J, Cifuentes L. Malformaciones congénitas en Chile y Latino América: Una visión epidemiológica del ECLAMC del período 1995-2008. Rev Med Chile. 2011;139:72-8.

12. Kucik J, Shin M, Siffel C, Marengo L, Correa A, for the Congenital Anomaly Multistate Prevalence and Survival Collaborative. Trends in Survival Among Children With Down Syndrome in 10 Regions of the United States. Pediatrics. 2013;131:e27-36.

13. Nazer J, Antolini MJ, Eugenia M, et al. Prevalencia al nacimiento de aberraciones cromosómicas en el Hospital Clínico de la Universidad de Chile: Período 1990-2001. Rev Med Chile. 2003;131:651-8.

14. Lizama M, Cerda J, Monge M, et al. Morbimortalidad hospitalaria en niños con síndrome de Down. Rev Chil Pediatr. 2016;87:102-9.

15. Milad M, Novoa JM, Fabres J, Samamé MM, Aspillaga C. Recomendación sobre Curvas de Crecimiento Intrauterino. Rev Chil Pediatr. 2010;81:264-274

16. Alsafadi TR, Hashmi S, Youssef H, Suliman A, Abbas H, Albaloushi M. Polycythemia in neonatal intensive care unit, risk factors, symptoms, pattern, and management controversy. J Clin
Neonatol. 2014;3:93.

17. AAP.Subcommittee on Hyperbilirubinemia. Management of Hyperbilirubinemia in the Newborn Infant 35 or More Weeks of Gestation. Pediatrics. 2004;114(1):297-316.

18. National Collaborating Centre for Women's and Children's Health Commissioned by the National Institute for Health and Clinical Excellence. Clinical Guideline: Neonatal jaundice. Royal College of Obstericians and Gynaecologists; 2010.

19. Adamkin DH. Neonatal hypoglycemia. Semin Fetal Neonatal Med. 2017;22:3641.

20. Lucas A, Morley R, Cole TJ. Adverse neurodevelopmental outcome of moderate neonatal hypoglycaemia. BMJ. 1988;297:1304-8.

21. Ministerio de Salud de Chile (MINSAL) Normas para el óptimo desarrollo de programas de búsqueda masiva de Fenilquetonuria (PKU) e Hipotiroidismo Congénito (HC) y otros Errores Innatos del Metabolismo (EIM). Santiago, Chile: Ministerio de Salud de Chile (MINSAL); 2007.

22. Guía Clínica AUGE: Síndrome de Dificultad Respiratoria en el recién nacido. $2^{\text {a }}$ ed. Santiago Chile: Ministerio de Salud de Chile (MINSAL); 2011. (Serie Guías Clínicas MINSAL).

23. Christiansen R. Hematologic Problems of the Neonate. $1^{\text {a }}$ ed. W.B. Saunders; 2000.

24. Moss AJ, Allen HD, editores. Moss and Adams' Heart disease in infants, children, and adolescents: including the fetus and young adult. 7th ed. Philadelphia: Wolters Kluwer Health/Lippincott Williams \& Wilkins; 2008. 2 p.

25. Gili JA, Poletta FA, Giménez LG, et al. Descriptive analysis of high birth prevalence rate geographical clusters of 
congenital anomalies in South America: Clusters of Congenital Anomalies in South America. Birt Defects Res A Clin Mol Teratol. 2016;106:257-66.

26. Ministerio de Salud de Chile (MINSAL). Guía Clínica Prevención Parto Prematuro. $2^{\mathrm{a}}$ ed. Santiago Chile: Ministerio de Salud de Chile (MINSAL); 2010.

27. Martin T, Smith A, Breatnach CR, et al. Infants Born with Down Syndrome: Burden of Disease in the Early Neonatal Period. J Pediatr. 2018;193:21-6.

28. Ergaz-Shaltiel Z, Engel O, Erlichman I, Naveh Y, Schimmel MS, Tenenbaum A. Neonatal characteristics and perinatal complications in neonates with Down syndrome. Am J Med Genet A. 2017;173:1279-86.

29. Sparks TN, Griffin E, Page J, Pilliod R, Shaffer BL, Caughey AB. Down syndrome: perinatal mortality risks with each additional week of expectant management: Perinatal mortality risk with Down syndrome. Prenat Diagn. 2016;36:368-74.

30. Mircher C, Toulas J, Cieuta-Walti $\mathrm{C}$, et al. Anthropometric charts and congenital anomalies in newborns with Down syndrome. Am J Med Genet A. 2017;173:2166-75.

31. Dolan SM, Gross SJ, Merkatz IR, et al. The Contribution of Birth Defects to Preterm Birth and Low Birth Weight: Obstet Gynecol. 2007;110:318-24.

32. Honein MA, Kirby RS, Meyer RE, et al. The Association Between Major Birth Defects and Preterm Birth. Matern Child Health J. 2009;13:164-75.
33. Malassine A, Frendo J-L, Evain-Brion D. Trisomy 21- affected placentas highlight prerequisite factors for human trophoblast fusion and differentiation. Int J Dev Biol. 2010;54:475-82.

34. Flöck A, Remig I, Müller A et al. Conflicting umbilical artery Doppler findings in fetuses with trisomy 21 . Arch Gynecol Obstet. 2015;292:613-7.

35. Jaruratanasirikul S, Limpitikul W, Dissaneevate P, Booncharoen P, Tantichantakarun P. Comorbidities in Down syndrome live births and health care intervention: an initial experience from the birth defects registry in Southern Thailand. World J Pediatr. 2017;13:152-7.

36. Astete C, Youlton R, Castillo S, Be C, Daher V. Análisis clínico y citogenética en 257 casos de Síndrome de Down. Rev Chil Pediatr. 1991;62:99-102.

37. Loane M, Morris JK, Addor M-C, et al. Twenty-year trends in the prevalence of Down syndrome and other trisomies in Europe: impact of maternal age and prenatal screening. Eur J Hum Genet. 2013;21:27-33.

38. Ramírez RC, Nazer HJ, Cifuentes OL, Águila RA, Gutiérrez RR. Cambios en la distribución etaria de las madres en Chile y en el Hospital Clínico de la Universidad de Chile y su influencia en la morbimortalidad neonatal. Rev Chil Obstet Ginecol. 2012;77:183-9.

39. Pardo Vargas RA, Aracena M, Aravena T et al. Consenso de la Rama de Genética de la Sociedad Chilena de Pediatría sobre las anomalías congénitas de mal pronóstico vital (ACMPV). Rev Chil Pediatr.
2016;87:422-31.

40. Ministerio de Salud de Chile (MINSAL), Subsecretaría de Salud Pública. Norma Técnica Nacional: Acompañamiento y atención integral a la mujer que se encuentra en alguna de las tres causales que regula la ley 21.030. Santiago Chile: Ministerio de Salud de Chile (MINSAL); 2018.

41. Brodwall K, Greve G, Leirgul E, et al. The five-year survival of children with Down syndrome in Norway 1994-2009 differed by associated congenital heart defects and extracardiac malformations. Acta Paediatr. 2018;107:845-53.

42. Irving CA, Chaudhari MP. Cardiovascular abnormalities in Down's syndrome: spectrum, management and survival over 22 years. Arch Dis Child. 2012;97:326-30.

43. Stoll C, Dott B, Alembik Y, Roth M-P. Associated congenital anomalies among cases with Down syndrome. Eur J Med Genet. 2015;58:674-80.

44. Donoso E, Vera C. Aumento de la mortalidad infantil en niños con síndrome de Down: Chile 1997-2013. Rev Med Chile. 2016;144:1432-9.

45. Walker B. Últimos avances en el diagnóstico prenatal: DNA fetal libre en sangre materna. Contacto Científico [Internet]. enero de 2017;7(1). Disponible en: http://contactocientifico.alemana.cl/ ojs/index.php/cc/article/view/435.

46. Firth H, Hurst J. Down's Syndrome (trisomy 21). En: Oxford Desk Reference Clinical Genetics \& Genomics. 2a ed. Reino Unido: Oxford University Press; 2017. p. 666-8. 